\title{
Analyzing the Effect of Dimples on Wind Turbine Efficiency Using CFD
}

\author{
Arun K.K ${ }^{1}$, Navaneeth V.R ${ }^{1}$, Sam Vimal Kumar $S^{2}$, Ajay $R^{2}$ \\ Associate Professor' ${ }^{\text {, P.G.Student }}{ }^{2}$, \\ Department of Mechanical Engineering, Kumaraguru College of Technology, India. \\ Coimbatore, Tamil Nadu -641049, India.
}

\begin{abstract}
In this energy hungry world, energy efficient methods to increase the extraction of work from various sources of renewable energy is always appreciated. One such commonly used source is wind energy. Wind turbines and their aerodynamics are always subjected to constant research for increasing their efficiency which convert the abundant wind energy into usable electric energy. In this project, one such attempt to increase the efficiency is made through the changes in surface topology. Dimples on the other hand are very efficient in reducing drag as is it evident from the reduction of drag and increase in lift in golf balls. The predominant factors influencing the efficiency of the wind turbines are lift and drag which are to be maximised and minimised respectively. Surface of turbine blades are included with dimples of various sizes and arrangements and are analyzed using CFD to obtain an optimum combination, through which efficiency of wind turbines can be maximised.
\end{abstract}

Keywords: Renewable Energy, Wind Energy, Dimples, CFD.

\section{INTRODUCTION}

The current energy crisis and fossil fuel depletion has urged many countries to switch to other forms of renewable sources of energy, and sooner or later, the total world has to switch to renewable energy. Among various forms of renewable energy, hydel and wind energy plays vital role in energy generation because of their surplus availability and higher efficiencies of energy transformation when compared to other sources such as Tidal or Solar. Also, various researches are taking forms on increasing the efficiencies of wind and hydel energies further.

\section{A. Study on Wind Turbines}

Wind power is an important source of environmental-friendly energy and has become more and more important in the recent years. The amount of installed wind power is increasing every year and many nations have made plans to make large investments in wind power in the near future. High efficiency wind turbines are being designed on the pursuit of reaching the Betz limit of energy conversion. The analysis of Lanchester and Betz (1920) showed that the maximum extraction of energy possible from a turbine rotor is $59.3 \%$ of the incoming kinetic energy[1].

\section{B. Types of Wind Turbines}

There are many different types of wind turbines and they can be divided into two groups of turbines depending on the orientation of their axis of rotation, namely horizontal axis wind turbines (HAWTs) and vertical axis wind turbines (VAWTs). The general public associates wind turbines with HAWTs and are unaware of the several other technologies based on the VAWT. Vertical axis wind turbines are less frequently used (Savonius and Darrieus are the most common in the group)[2].

Based on the commercial benefits and better energy conversion, Horizontal axis wind turbines are the more preferred over the vertical axis wind turbines, and thus we take Horizontal axis wind turbines for this study.

\section{Working of Wind Turbines}

Wind turbines work by turning the kinetic energy of the wind into torque (a force) that causes the wind turbine to turn and drives an electrical generator. The wind is made up of real matter with mass, when mass is moving it has kinetic energy. As the wind causes the wind turbine to turn, we are absorbing the energy in the wind. The energy that is absorbed from the wind is converted into mechanical energy that is used to drive an electrical generator and then converted into electrical energy[3].

Airflow over any surface creates two types of aerodynamic forces - drag forces, in the direction of the airflow, and lift forces, perpendicular to the airflow as shown in Figure 1. Either or both of these can be used to generate the forces needed to rotate the blades of a wind turbine.

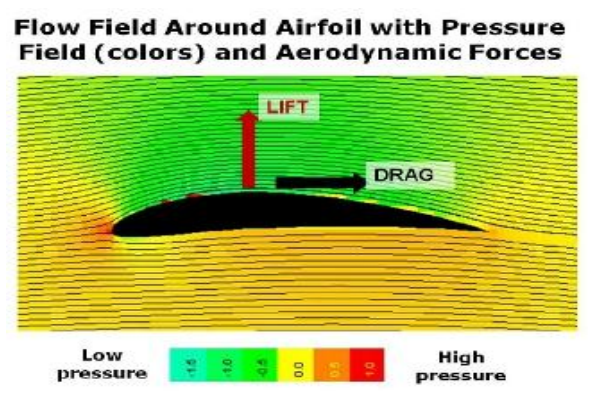

Figure 1: Varying pressure field over an airfoil

Of these two forces that influence the rotation of a wind turbine, lift is the one that influences the horizontal axis wind turbines in a positive manner, helping in energy conversion to a greater extent. Measures have to be taken in order to maximise the effect of lift for optimum energy conversion. On the other hand, all moving objects in air experience an opposing force which is called drag. Drag reduces degree of energy conversion and hence, methods that minimise drag effect on wind turbines would be appreciated. 


\section{Dimples}

Dimples are small depressions on the surface which is usually permanent like in case of a golf ball. The presence of dimples increases the amounts of energy and linear momentum that are available to those particles of fluid that move in a tiny region near the surface of the ball called the boundary layer as shown in

Figure 2. The existence and importance of the boundary layer are due to the peculiar behaviour of fluid friction near the surface of a solid body that is in relative motion with the surrounding fluid. In the case of a golf ball, dimples trip air particles that are moving close to them; this disturbance causes the particles to jiggle sideways while they travel forward, instead of staying in lanes of traffic that are perfectly parallel to each other at all times, as expected in laminar flow. This jiggling forces particles in adjacent lanes to bump into each other, causing linear momentum to be transferred through bumping. Particles that were moving slowly gain a little more speed; those that were moving fast lose a little speed in the process.

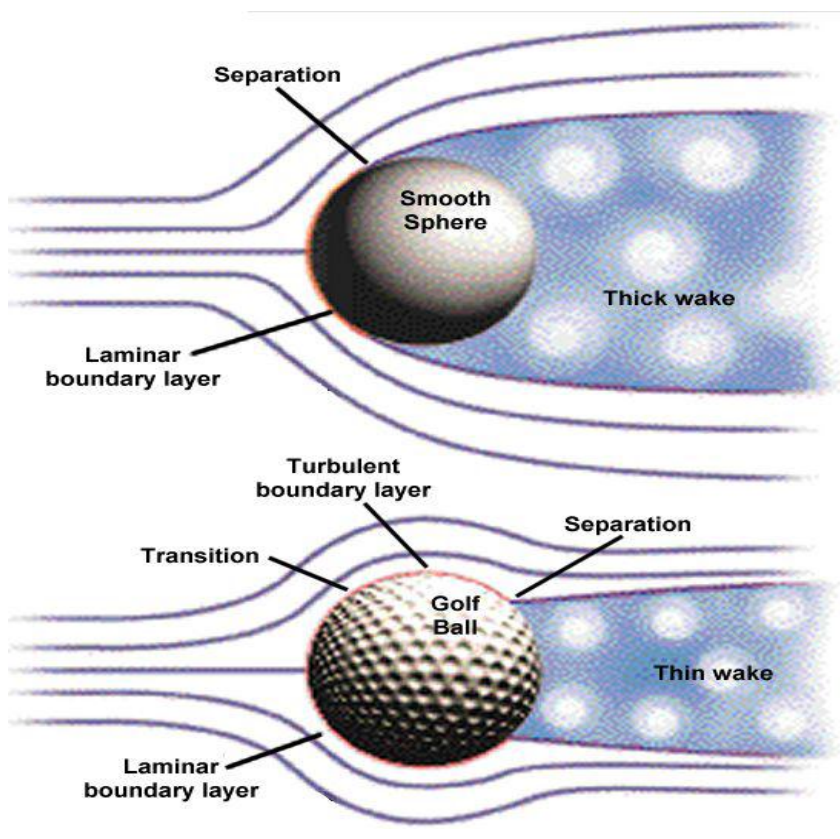

Figure 2: Effects of dimples on golf ball

Among other results in introducing dimples, it indicates two relevant things, namely:

- The changes in velocity noted earlier increase both the kinetic energy and the linear momentum of the whole flow within the boundary layer;

- Turbulent flow has more kinetic energy and more linear momentum than laminar flow. When bumping is vigorous enough, its net result is that the bulk flow of air in the boundary layer becomes turbulent.

\section{METHODOLOGY AND APPROACH}

\section{A. Turbine Blade Design Methodology}

Table 1: Airfoil position data[7].

\begin{tabular}{|c|c|c|c|c|c|}
\hline Span / r & Span (m) & $\begin{array}{l}\text { Twist } \\
\text { (deg) }\end{array}$ & Chord / $\mathrm{r}$ & Chord (m) & Airfoil \\
\hline 0.075 & 3 & 42 & 0.0614 & 2.456 & \multirow[t]{8}{*}{ S818 } \\
\hline 0.125 & 5 & 32 & 0.0683 & 2.7304 & \\
\hline 0.175 & 7 & 23 & 0.0745 & 2.9808 & \\
\hline 0.225 & 9 & 15 & 0.0778 & 3.1128 & \\
\hline 0.275 & 11 & 11.5 & 0.0754 & 3.0172 & \\
\hline 0.325 & 13 & 8.2 & 0.0719 & 2.8752 & \\
\hline 0.375 & 15 & 7 & 0.0683 & 2.7328 & \\
\hline 0.425 & 17 & 6 & 0.0648 & 2.5916 & \\
\hline 0.475 & 19 & 5 & 0.0613 & 2.4504 & \multirow[t]{8}{*}{ S825 } \\
\hline 0.525 & 21 & 4 & 0.0613 & 2.4504 & \\
\hline 0.575 & 23 & 4.15 & 0.0542 & 2.166 & \\
\hline 0.625 & 25 & 3.85 & 0.0506 & 2.0248 & \\
\hline 0.675 & 27 & 3.25 & 0.0471 & 1.8828 & \\
\hline 0.725 & 29 & 2.75 & 0.0436 & 1.744 & \\
\hline 0.775 & 31 & 1.25 & 0.0402 & 1.6096 & \\
\hline 0.825 & 33 & 0.75 & 0.037 & 1.4816 & \\
\hline 0.875 & 35 & 0.55 & 0.0339 & 1.354 & \multirow[t]{4}{*}{ S826 } \\
\hline 0.925 & 37 & 0.85 & 0.0307 & 1.2264 & \\
\hline 0.975 & 39 & 0.05 & 0.0275 & 1.0988 & \\
\hline 1 & 40 & 0 & 0.0242 & 0.9696 & \\
\hline
\end{tabular}

Before we could proceed to applying the idea of drag reduction using dimples, there are a number of other factors to be considered like the turbine blade profile, the angle of approach, type of turbine, type of rotor, the analysis technique and many more. Based on the purpose and our requirement, our airfoil blades are selected from the NREL S-Series, which are found to be thicker than the ones that are seen on airplanes. Also, based on our requirements for the generation of $1.5 \mathrm{MW}$ power, at wind speeds of $12-20 \mathrm{~m} / \mathrm{s}$, the optimal blade was found to have a span of 40 meters[4].

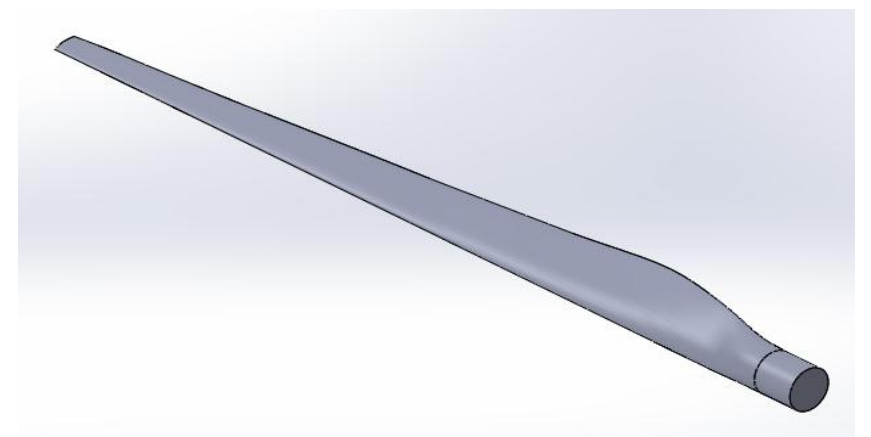

Figure 3: Wind turbine blade model 
The blade is joined with the hub with the help of a cylindrical root section. The blade profile is divided into three sections. Three different profiles such as S818, S825, S826[5] are included in each section of the blade, starting from the root. The blade is modelled with Solidworks CAD package. Airfoil are modelled with the point data which defines a particular airfoil. The blade dimensions including twist, chord length, span with respect to the blade length of $40 \mathrm{~m}$ are given in above table[6].

\section{B. Simulation and Analysis Methodology}

There is a requirement for prediction methodologies that are capable of addressing the in-situ performance of multiple turbine installations within a specific local environment and operating in a range of conditions. Earlier the scientist used to create scaled prototypes of the aerofoil to be analyzed and them place them in large wind tunnels to simulate the real world working conditions[8]. Sensors were placed at different points in the tunnel as well as the object of study to obtain, tabulate and analyze the data. This method was costly and time consuming and not as accurate as most of the times the scaled models behaved differently from the actual sized products.

However in the resent modern times this process of testing and analysis has been highly computerized and now is solved using Computational Fluid Dynamics (CFD)[9] which is a branch of fluid mechanics which uses numerical analysis and algorithms to solve and analyze problems that involve fluid flows. Computers are used to perform the calculations required to simulate interactions of liquids and gases with surfaces defined by boundary conditions. In all the approaches a same basic procedure is followed

- $\quad$ The geometry of the problem is defined.

- The volume occupied by the fluid is divided into discrete cells (uniform or non-uniform) by a process called meshing.

- The physical modeling is defined like the equations of flow etc.

- Boundary conditions are defined which involves specifying fluid behavior and properties at the boundaries of the problem.

- The simulation is started and the equations are solved iteratively.

- Finally a postprocessor is used for the analysis and visualization of the resulting solution.

The geometry of the problem here is the turbine blade which has to be analysed. The blade is modelled using the solid works modelling software and then imported to the CFD analysis software. Here a domain[10] is created for the study which acts as a virtual boundary for the fluid.

The next step that is meshing. The accuracy of any simulated output is directly dependent on the resolution of the meshed model i.e. the smaller the mesh element size, the higher the number of elements and so more accurate will be the result.

The boundary conditions then need to be defined. Now, there are a number of ways by which the boundary conditions of a wind turbine can be defined but among all the most commonly used one is the actuator disc model. Although this model is not the best, it is an optimum balance between accuracy and computational effort.

\section{Actuator Disk Principle}

The function of a wind turbine rotor is to extract the kinetic energy of the incoming flowfield. A thrust in the direction of the incoming flowfield is produced with a magnitude directly related to the change in kinetic energy. With the rotational movement and the frictional drag of the blades, the flowfield is furthermore imparted by a torque which contributes to the change in kinetic energy. Thus, the flowfield and forces related to operating wind turbine rotors are governed by the balance between the thrust and torque on the rotor and the kinetic energy of the incoming flowfield. The behaviour of a wind turbine rotor in a flowfield may conveniently be analyzed by introducing the actuator disc principle[11]. The basic idea of the actuator disc principle in connection with rotor aerodynamic calculations is to replace the real rotor with a permeable disc of equivalent area where the forces from the blades are distributed on the circular disc. The distributed forces on the actuator disc alter the local velocities through the disc and in general the entire flowfield around the rotor disc. Hence, the balance between the applied forces and the changed flowfield is governed by the mass conservation law and the balance of momentum, which for a real rotor is given by the axial and tangential momentum equations. The Figure 4 below shows the representation of an actuator disk.

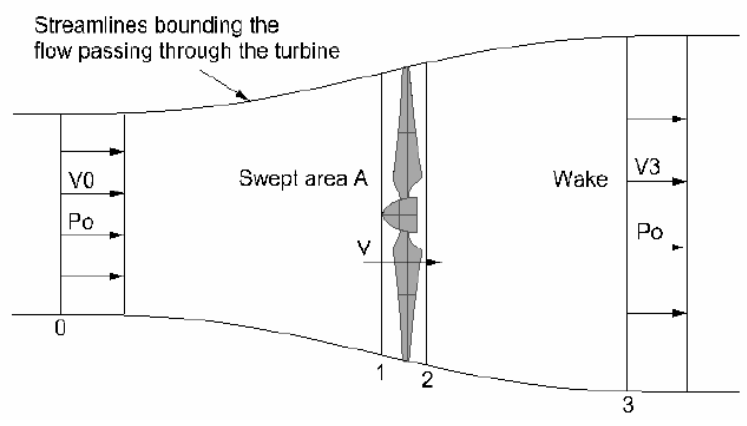

Figure 4: Representation of Actuator Disc Method

A real rotor, however, is never uniformly loaded as assumed by the Rankin-Froude actuator disc model, and in order to analyze the radial load variation along the blades, the flowfield is subsequently divided into radial independent annular stream tubes in the classical Blade Element Momentum, (BEM) method. The annual stream tubes are independent is one the basic assumption for the classical BEM method[12].

Thus by using this methodology along with the RANS (Reynolds Averaged Naiver-Stokes) equations a near to actual result can be obtained[13]. The RANS equations are chosen above the Direct Naiver-Stokes (DNS) equations because of their simplicity and significantly lesser computational time requirement. 


\section{RESULT AND DISCUSSION}

Analysis is proceeded in two steps. The first one without dimples which is done in order to find out the degree of accuracy to which obtained results and the actual results match and also to differentiate the efficiency between the blades with and without dimples. The second step is the analysis of the blade with dimples.

Blade alignment, domain dimensions, mesh settings and boundary conditions are considered to be the same for both the blades.

Since the blades of the wind turbine are identical, and are symmetrically arranged at angular gaps of 1200 due to symmetry, it is enough to analyse only one blade with a domain in the shape a sector having 1200 angle. The blade is positioned $1 \mathrm{~m}$ away from the axis of rotation to compensate for the hub.

The wind speed considered for analysis is $12 \mathrm{~m} / \mathrm{s}$ with the blade rotating at the speed of $2.2 \mathrm{rad} / \mathrm{s}$ or $21 \mathrm{RPM}$. The relative motion between the blade tip and the flow stream results in a relative velocity which approaches the blade at an angle of 960 (this angle is obtained by using the velocity vectors of the wind as well as the blade and then finding their resultant), So the blade is also rotated in order to make it's tip incline at the same angle. This causes the tip of the blade to have an almost zero angle of attack. A fluid domain is then created.

Based on the actuator disc principle, a fluid domain is created for the wind turbine with dimensions as follows:

Inlet face radius $=120 \mathrm{~m}$

Inlet face distance from rotor $=90 \mathrm{~m}$

Outlet face radius $=240 \mathrm{~m}$

Outlet face distance from rotor $=180 \mathrm{~m}$

The above-mentioned radii are that of the sector which will cover only one blade, using the symmetric boundary condition, the entire wind mill is analysed. The domain is created using The Ansys Design Modular software. The blade profile is then subtracted from the blade to create a void, which acts as a virtual boundary.

\section{A. Mesh Settings}

The mesh is generated using the following settings.

Table 2: Mesh details

\begin{tabular}{|l|l|}
\hline Sizing \\
\hline Use Advanced Size Function & On: Proximity and Curvature \\
\hline Relevance Centre & Medium \\
\hline Smoothing & Medium \\
\hline Transition & Slow \\
\hline Statistics & \multicolumn{2}{|l|}{} \\
\hline Nodes & 174725 \\
\hline Elements & 778753 \\
\hline Mesh Metric & Skewness \\
\hline Average & 0.23823 \\
\hline
\end{tabular}

\section{B. Boundary Conditions}

The fluid medium is set as air at room temperature. The inlet velocity of wind is given as $12 \mathrm{~m} / \mathrm{s}$. As this is an actuator disc model, a moving frame of reference is created, with axis of rotation about the $\mathrm{z}$-axis. The speed of rotation is given as 2.2 $\mathrm{rad} / \mathrm{s}$ (this speed is the maximum speed with which the windmill will rotate in its optimum conditions i.e. wind speeds of $12 \mathrm{~m} / \mathrm{s}-20 \mathrm{~m} / \mathrm{s}$ ). K-omega equation model is chosen for the analysis and hybrid initialization is done. The solution is run for 1500 iterations to converge to the result.

\section{Analysis Result}

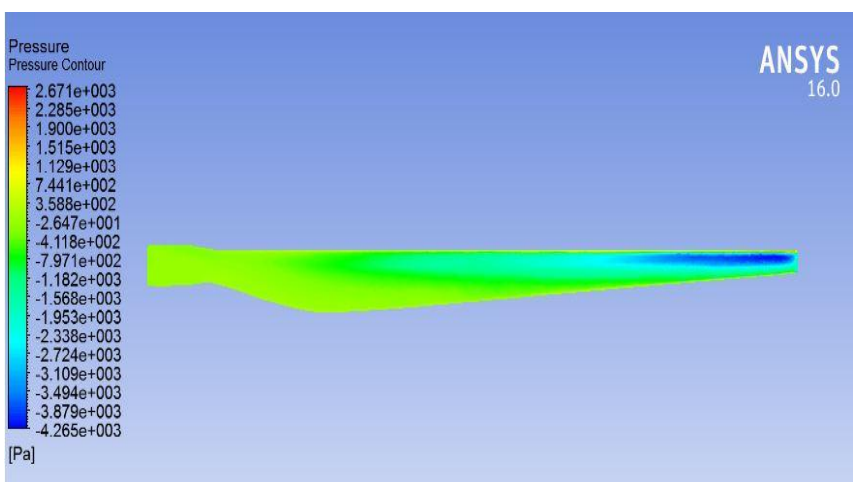

Figure 5: Top view of plain blade pressure field

The plain blade is first analysed with the above mentioned settings and the following results are obtained. The pressure contour of the top side of the blade is shown in the Figure 5 which shows the low pressure that is created at the top surface of the wind turbine blade.

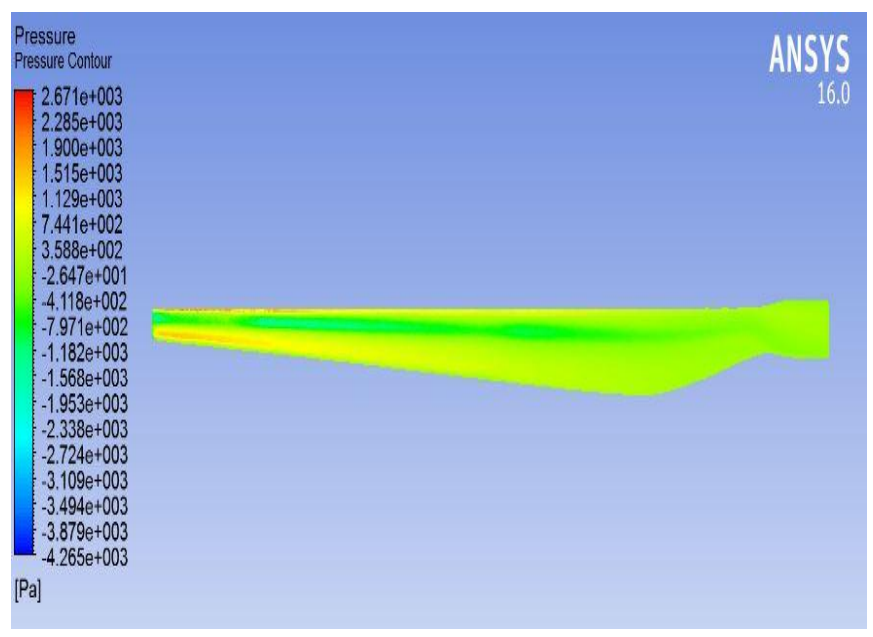

Figure 6: Bottom view of plain blade pressure field

The pressure contour of the bottom side of the blade is shown in the Figure 6 above. The pressure developed at the bottom is significantly higher when compared to that of the top side of the blade. This confirms that lift is generated. 


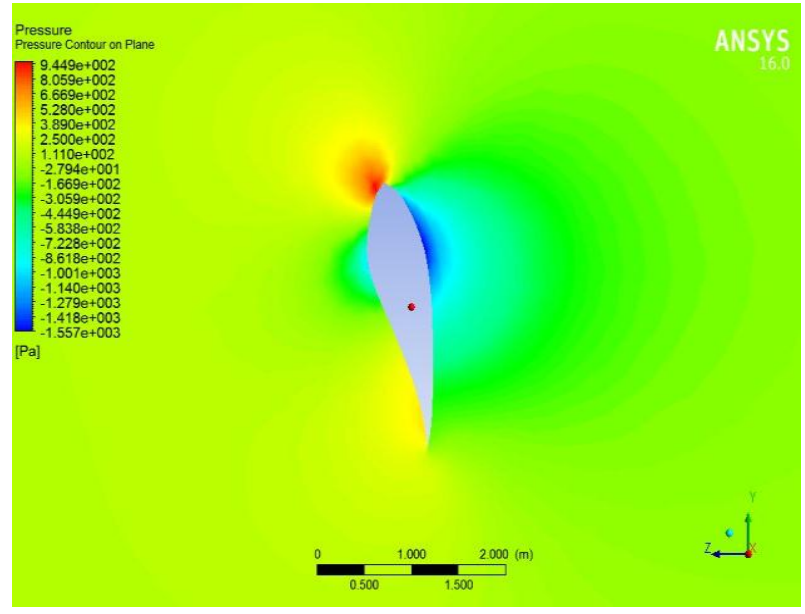

Figure 7: Cross section of plain blade pressure field

From the above Figure 7 it is clear that a high pressure is created at the bottom surface just near the leading edge of the blade. This high pressure creates a resistance to the rotation of the turbine and is otherwise known as drag. So, from this the position at which the drag force acts and is maximum is visible.

This data is then used to approximate a region where the dimples would be most effective and this region is found to be on the bottom face of the blade in the mid region near the trailing edge. Only the mid region is selected as the region near the root is too slow for drag to have a considerable effect and the region near the tip is too fast for dimples to have any considerable effect. The dimples are thus designed as shown in the Figure 8 below.

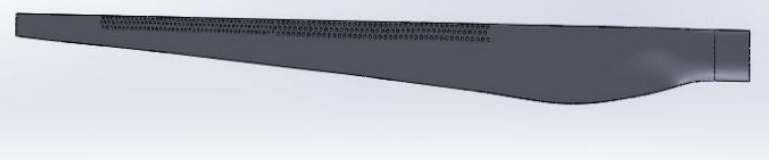

Figure 8: Revised blade design with Dimples

After the dimples have been included in the design of the blade, the dimpled blade is then analyzed with the above mentioned settings and the following results are obtained.

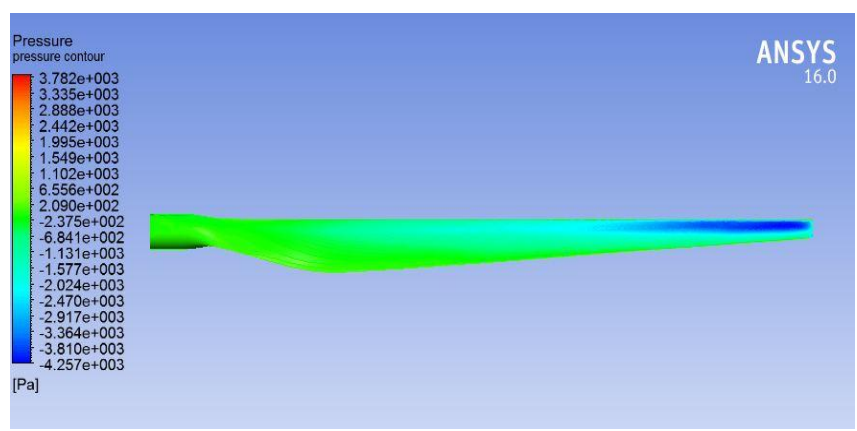

Figure 9: Top view of dimpled blade pressure field
The pressure contour of the top side of the dimpled blade is shown in the Figure 9 which shows that the low pressure that is created at the top surface of this blade is similar to that of the plain blade. This implies that there is no effect of the dimples on the pressures of the top surface.

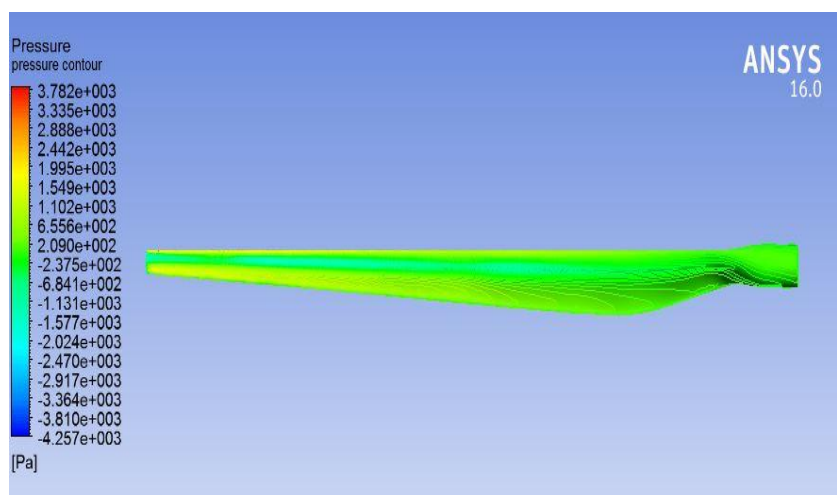

Figure 10: Bottom view of dimpled blade pressure field

The pressure contour of the bottom side of the dimpled blade is shown in the Figure 10 above. The pressure developed at the bottom is significantly higher when compared to that of the top side of the blade. This high pressure region however is much more lesser compared to the plain blade at the region where the dimples have been placed. This indicates that the dimples have effectively reduced the pressure in the region by creating turbulence and hence reduced the drag.

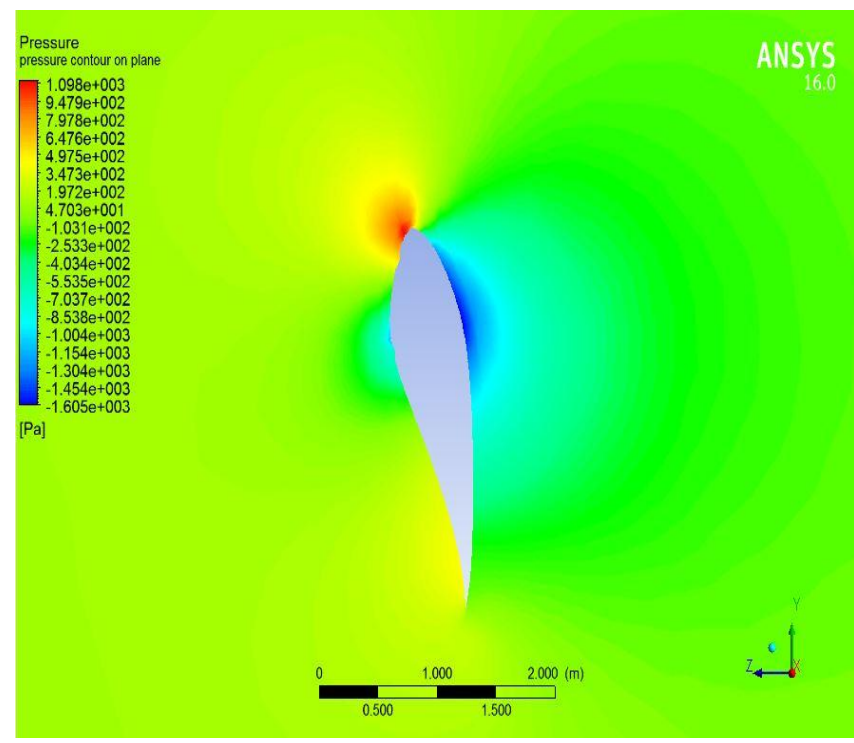

Figure 11: Cross section of dimpled blade pressure field

By comparing the pressure contour of the cross-section of the dimpled blade as shown in the above figure to that of the plain blade, it is seen that the highest pressure as described in the legends have a negligible difference but the region of high pressure is significantly larger in the plain blade when compared to the dimpled one. This implies that due to a smaller region of high pressure, the drag in dimpled blades is lesser. 


\section{Torque and Power}

Using the function calculator of Ansys CFD Post, the torque produced by the wind turbine is calculated. Only the torque produced by the blade is calculated about the global z-axis as it is the defined axis of rotation in this model.

For the plain blade, the torque produced by single blade is found to be $258940 \mathrm{Nm}$ and so the torque produced by the turbine is $776820 \mathrm{Nm}$ which can be converted to power using the formula,

$$
\mathrm{P}=2 \pi \mathrm{NT} / 60
$$

Where

$\mathrm{P}$ is the power,

$\mathrm{N}$ is the seed of rotation in RPM and

$\mathrm{T}$ is the torque produced.

The power is thus found to be $1.70 \mathrm{MW}$. This power is transmitted to the generator and during the transmission, a loss of $20 \%$ occurs[14]. This makes a power of $1.36 \mathrm{MW}$ to the generator which is much closer to the actual value. For the dimpled blade the torque produced by single blade is found to be $296704 \mathrm{Nm}$ and so the torque produced by the turbine is $890112 \mathrm{Nm}$ which when converted to power is $1.95 \mathrm{MW}$. After the transmission loss, the power available to the generator is $1.56 \mathrm{MW}$.

While comparing the power outputs, it is inferred that there is a $14.7 \%$ increase of power in dimpled blade turbine when compared to the plain blade turbine.

\section{CONCLUSION}

The objective of the study is to analyze the performance characteristics of a wind turbine designed with dimples for the purpose of reducing drag. This is an attempt to increase the efficiency of the wind turbines. The motivation in using dimples in order to modify the surface topology of wind turbines is the functional advantage they provide evident from the golf balls. The study focuses on the aerodynamic effects which resulted due to the inclusion of dimples, contributing to torque and eventually improved performance levels.

The blade profile is chosen from the NREL S series, combining 3 profiles together for optimum performance of generating about 1.5 MW power under wind speed range of $12-20 \mathrm{~m} / \mathrm{s}$. The combination is proved to have more strength and is found thicker than most of other commercially available wind turbine blades. With promising accuracy from actuator disc method, preliminary analysis is made without any modifications on the surface, providing all the required boundary conditions. Nearly $1.36 \mathrm{MW}$ power was found to be generated and with high levels of accuracy on comparison with actual values, we proceeded towards the inclusion of dimples. Based on the pressure contour obtained, dimples are included on the high pressure bottom side of the turbine. Further analysis with dimples have increased the performance to a greater extent, about $1.56 \mathrm{MW}$ power was found to be generated, a $14.5 \%$ increase in performance, to be precise. Thus, inclusion of dimples on the surface of wind turbine blades will increase performance levels.

\section{REFERENCES}

[1] A. . Hansen and C. . Butterfield, "Aerodynamics of horizont al-axis wind turbines," 1993.

[2] M. O. L. Hansen, J. N. Sørensen, S. Voutsinas, N. Sørensen, and H. A. Madsen, "State of the art in wind turbine aerodynamics and aeroelasticity," 2006.

[3] O. D. Vries, "On the Theory of the Horizontal-Axis Wind Turbine," 1983.

[4] A. Crespo, J. Hernandez, and S. Frandsen, "Survey of modelling methods for wind turbine wakes and wind farms.," 1999.

[5] F. Bertagnolio, F. Bertagnolio, N. N. Sørensen, N. N. Sørensen, J. Johansen, and J. Johansen, "Profile catalogue for airfoil sections based on 3D computations," 2006.

[6] N. Hoyle and E. Flow, "Royal Academy of Engineering : Renewable Power," 2009.

[7] P. J. S. and R. J. Crossley, "Wind Turbine Blade Design," 2012.

[8] P. R. Ebert and D. H. Wood, "The near wake of a model horizontal-axis wind turbine-I. Experimental arrangements and initial results," 1997.

[9] C. Van Dam, D. Chao, and D. Berg, "CFD analysis of rotating two-bladed flatback wind turbine rotor.," 2008.

[10] "Energies_Free Full-Text _ CFD in Wind..pdf."

[11] [R. Flemming and J. Nørkær, “Actuator Disc Methods Applied to Wind Turbines by Robert Mikkelsen Dissertation submitted to the Technical University of Denmark in partial fulfillment of," 2004.

[12] E. Benini and A. Toffolo, "Optimal Design of Horizontal-Axis Wind Turbines Using Blade-Element Theory and Evolutionary Computation," 2002.

[13] D. Hartwanger and A. Horvat, "3D modelling of a wind turbine using CFD," 2008.

[14] A. Sarkar and D. K. Behera, "Wind Turbine Blade Efficiency and Power Calculation with Electrical Analogy," 2012. 